\title{
Highly Efficient and Environmentally Stable \\ Flexible Color Converters based on Confined
}

\section{$\mathrm{CH}_{3} \mathrm{NH}_{3} \mathrm{PbBr}_{3}$ Nanocrystals}

Andrea Rubino, Miguel Anaya†, Juan F. Galisteo-López, T. Cristina Rojas, Mauricio E. Calvo* and Hernán Míguez*

Institute of Materials Science of Seville, Spanish National Research Council-University of Seville, C/Américo Vespucio 49, 41092, Seville, Spain.

KEYWORDS. Perovskites, nanocrystals, quantum confinement, quantum yield, flexible film, color converter.

ABSTRACT. In this work, we demonstrate a synthetic route to attain methylammonium lead bromide $\left(\mathrm{CH}_{3} \mathrm{NH}_{3} \mathrm{PbBr}_{3}\right)$ perovskite nanocrystals (nc-MAPbBr $3,1.5 \mathrm{~nm}<$ size $<3 \mathrm{~nm}$ ) and provide them with functionality as highly efficient flexible, transparent, environmentally stable and adaptable color converting films. We use nanoparticle metal oxide (MOx) thin films as porous scaffolds of controlled nanopore size distribution to synthesize nc-MAPbBr3 through the infiltration of perovskite liquid precursors. We find that the control over the reaction volume imposed by the nanoporous scaffold gives rise to a strict control of the nanocrystal size, which allows us to observe well defined quantum confinement effects on the photoemission, being the 
luminescence maximum tunable with precision between $\lambda=530 \mathrm{~nm}$ (green) and $\lambda=490 \mathrm{~nm}$ (blue). This hybrid nc-MAPbBr $3 / \mathrm{MOx}$ structure presents high mechanical stability and permits subsequent infiltration with an elastomer to achieve a self-standing flexible film, which not only maintains the photoemission efficiency of the nc-MAPbBr3 unaltered, but also prevents their environmental degradation. Applications as adaptable color converter layers for light emitting devices are envisaged and demonstrated.

\section{Introduction}

One of the most appealing applications of organic metal halide perovskites lies in the field of light emitting devices (LEDs), where they can be used as electro- or photo-luminescent materials. ${ }^{1-5}$ In this context, one of the most attractive emitters is methylammonium lead bromide ( $\mathrm{MAPbBr} 3)$, as it presents an efficient emission centered in the green region of the visible spectrum. ${ }^{6}$ In fact, due to their high photoluminescence (PL) quantum yield (QY), these materials offer the interesting possibility to be implemented as color converting layers within a LED. A color converter should fulfill a series of requirements, such as: i) possessing a high PL QY, ii) being amenable to be used as large area (squared centimeters) coatings, iii) being adaptable to curved surfaces, and iv) being stable against environmental changes. ${ }^{7}$ Also desirable would be v) to have a means of tuning their emission in a wide visible frequency range and, aiming at displays, vi) to be able to process them as transparent films. ${ }^{8}$ Considering all this, perovskite nanocrystals, and more specifically nc$\mathrm{MAPbBr}_{3}$, stand out as promising candidates for a new generation of color converting coatings for LEDs. Since a synthetic route was first proposed to attain colloidal nc-MAPbBr $3,{ }^{9}$ a great effort has been made to improve their QY, ${ }^{10,11}$ values as high as $99 \%$ in solution being achievable nowadays. ${ }^{12}$ However, as it has been recently pointed out by Sargent et al., ${ }^{13}$ the intense 
photoluminescence (PL) of colloidal dots dispersed in liquid media is quenched when they are shaped as solid-state films, which is indispensable to use them in optoelectronic devices. Other approaches that make use of porous particles ${ }^{14-16}$ or films ${ }^{17-19}$ that act as matrices to grow $\mathrm{ABX}_{3}$ nanocrystals give rise to materials that either lack optical quality, as a result of the large size and aggregation of the host particles, or that are not suitable to create flexible and adaptable structures, thus hindering their use as coatings.

In this work, we demonstrate a synthetic pathway to attain large area, flexible, adaptable, transparent, wavelength tunable and environmentally stable highly efficient (QY>50\%) light emitting films based on nc-MAPbBr 3 , hence fulfilling all requisites to be considered as suitable candidates as color converters for LEDs. By dispersing the perovskite precursors in a nanopore size controlled optical quality metal oxide (MOx) host, we prevent aggregation and hence PL quenching. As the emission is governed by strong quantum confinement effects, control over the average nanocrystal size synthesized within the porous network yields a tunable emission from green to blue. Finally, infiltration of nc-MAPbBr 3 is carried out in such a way that leaves room for a subsequent infiltration of an elastomer into the porous network, which allows detaching it from the substrate. This process leads to a flexible and elastic self-standing version of the original coating that both preserves the high luminescence of nc-MAPbBr 3 and is capable of resisting the presence of moisture in the environment.

\section{Result and discussion}

Nanoporous films, made by dip coating flat substrates in MOx colloidal suspensions with narrow particles size distribution and average diameter in the range of the few tens of nanometers, are used as hosts to infiltrate $\mathrm{MAPbBr} 3$ precursors and obtain embedded nc-MAPbBr3. The use of nanoparticles with sizes well below visible light wavelengths prevents diffuse scattering and thus 
assures transparency of the porous film. ${ }^{20}$ The voids between the particles of these MOx layers constitute around $35-40 \%$ of the total volume of the film and are fully accessible from the top of the structure..$^{21,22}$ Examples of the detailed structural characterization, carried out using porosimetry and X-ray diffraction (XRD), of host matrices made of $\mathrm{SiO}_{2}$ nanoparticles of $30 \mathrm{~nm}$ average diameters is provided as Supplementary Information (see Figure S1). Then, MAPbBr3 precursors are infiltrated by spin coating and, after thermal annealing, nanocrystals are attained. The infiltration of $\mathrm{MAPbBr}_{3}$ precursors is carefully carried out to avoid the presence of a capping layer on top, hence ensuring that the photoemission comes exclusively from the perovskite synthesized within the pores. This is confirmed by cross-sectional FESEM back scattered electron images as well as by EDX analysis of the cross section of an $\mathrm{ABX}_{3}$ infiltrated $\mathrm{SiO}_{2}$ film. The combination of both techniques reveals bright spots corresponding to $\mathrm{Z}$-heavier $\mathrm{Pb}$ atoms of $\mathrm{ABX} \mathrm{X}_{3}$ perovskite randomly distributed within the porous $\mathrm{SiO}_{2}$ layer and the absence of an overlayer of bulk perovskite, as also shown in Figure S1. The results of the microstructural and elemental analysis of a cross section of the $\mathrm{MAPbBr}_{3} @ \mathrm{SiO}_{2}$ ensemble performed by transmission electron microscopy (TEM) permits to identify the spherical $\mathrm{SiO}_{2}$ particles forming the stack (Figure 1a) as well as, zooming in, the large amount of smaller nanocrystals deposited onto them (Figure $1 \mathrm{~b}$ and c). Samples were cleaved using a focused ion beam. An even closer analysis by high resolution TEM imaging of these particles (Figure 1d) reveals a crystalline structure with an interatomic distance of $0.22 \mathrm{~nm}$ that can be assigned to the $[2,2,0]$ family of planes associated to the tetragonal phase of the $\mathrm{MAPbBr} 3$ perovskite. Elemental analysis maps of $\mathrm{Si}, \mathrm{Pb}$ and $\mathrm{Br}$ obtained by $\mathrm{EDX}$ spectroscopy (Figures 1f, 1g, 1h) confirms the presence of nanocrystals exclusively within the pore network. Indeed, $\mathrm{Si}$ maps reproduced the morphology of the $\mathrm{SiO}_{2}$ nanoparticles, whereas darker regions correspond to the pore network. Complementarily, $\mathrm{Pb}$ and $\mathrm{Br}$ signals are preferentially 
detected within the pores of the structure. The average $\mathrm{MAPbBr} 3$ nanocrystal size was adjusted by controlling the deposition parameters, mainly the concentration of precursors, average diameters ranging from $1.8 \mathrm{~nm}$ to $2.3 \mathrm{~nm}$, attained for the lowest ( $5 \mathrm{wt} . \%$ ) and highest (40 wt.\%) precursor solution concentrations employed, respectively (Figure S1d). The increase of the average crystal size with the precursor concentration was confirmed by measuring grazing wide angle X-ray diffraction (GIWAX) of a series of $\mathrm{SiO}_{2}$ nanoparticle layers infiltrated by different concentrations of $\mathrm{MAPbBr}_{3}$ precursors. In all cases, the diffractograms reveal the presence of $\mathrm{MAPbBr}_{3}$ perovskite peaks (shown in Figure S1b) and a gradual narrowing of the 110 peak $\left(2 \operatorname{theta}=14.09^{\circ}\right.$ ) as the concentration of the precursors increases. In order to confirm the generality of the method herein proposed, infiltration experiments were carried out using $\mathrm{CH}_{3} \mathrm{NH}_{3} \mathrm{PbI}_{3}\left(\mathrm{MAPbI}_{3}\right)$ as well as $\mathrm{MAPbBr} 3$ precursors within other metal oxide nanoparticle host matrices such as $\mathrm{TiO}_{2}(12 \mathrm{~nm}$ particle size), $\mathrm{SnO}_{2}(8 \mathrm{~nm}$ particle size $)$ or $\mathrm{SiO}_{2}$ of a different particle size $(10 \mathrm{~nm})$, with similar results in all cases.

Since the porous $\mathrm{SiO}_{2}$ layers used as hosts are transparent in most of the ultraviolet (UV) range and completely transparent in the visible region (see figure S2), the photoluminescence (PL) of embedded nc-MAPbBr3 can be photoexcited using any source within that range. In Figure 2, the emission spectra of nanocrystals obtained from solutions containing different $\mathrm{MAPbBr} 3$ precursor concentrations and photoexcited using a source of $\lambda=450 \mathrm{~nm}$ are shown. The spectral position of the emission maximum shifts gradually to higher energies as the precursors concentration decreases (Figure 2a), in good agreement with the GWAX characterization results. Strong PL blue shifts, with respect to the bulk material emission, are observed for all nc-MAPbBr3 and nc-MAPbI 3 synthesized in the different MOx matrices tested, as displayed in Figure S3. For the lowest concentration employed ( $2 \mathrm{wt} . \%$ ), nanocrystals present an emission peak centered at $\lambda_{\max }=504 \mathrm{~nm}$ 
or at $\lambda \approx 495 \mathrm{~nm}$ for hosts made, respectively, of $30 \mathrm{~nm}$ and $10 \mathrm{~nm} \mathrm{SiO} 2$ nanoparticles (Figure S3a). These correspond to energy shifts of $\Delta \mathrm{E}=0.155 \mathrm{eV}$ and $\Delta \mathrm{E}=0.200 \mathrm{eV}$ relative to a layer of bulk $\operatorname{MAPbBr} 3\left(\lambda_{\max }=538 \mathrm{~nm}\right)$. The strong spectral shift of these peaks may be related to a good extent to quantum exciton confinement effects that occur in the as-synthesized nc-MAPbBr3, since the estimated exciton Bohr radius is around $3 \mathrm{~nm} .{ }^{23}$ In addition, the full width at half maximum of the PL peak is lower than $30 \mathrm{~nm}$ for all $\mathrm{MAPbBr}_{3}$ nanocrystal sizes, which is comparable to those obtained in colloidal systems. Accordingly, a similar displacement of the photoexcitation edge can be seen in Figure 2b. The sharp edge near the photoexcitation threshold confirms the narrow nanocrystal size distribution achieved in each infiltrated $\mathrm{SiO}_{2}$ nanoparticle scaffold, also corroborated by the displacement of the absorption edge (Figure S4).

The fine control of nc-MAPbBr 3 size by the concentration of the precursors infiltrated within the porous network can be rationalized in terms of the rates at which the different processes involved take place in the matrix during spin-coating. In a single heterogeneous phase, in absence of protective surface agents, the nucleation and growth are dictated by the elimination of the solvent. At the initial stage of the deposition step, before spinning starts, the infiltration rate of the $\mathrm{SiO}_{2}$ porous matrix is much faster than the solvent evaporation as a consequence of the low vapor pressure of the N,N-dimethylformamide. This yields a homogeneous distribution of $\mathrm{MAPbBr}_{3}$ precursors all along the film, hence favouring a uniform embedding of nc- $\mathrm{MAPbBr}_{3}$ for the range of concentrations analyzed in our experiments. ${ }^{24}$ Once the spin coating process begins, the rapid elimination of the solvent promotes the formation of a large amount of nuclei and prevents the formation of large perovskite particles as a consequence of the super-saturation of precursors in the solvent. ${ }^{25}$ In our case, the concentration range leads to particle size in the range of 1.5-3.0 nm. At higher precursor concentrations (15wt.\%-40wt.\%), the growth is not limited as much by 
diffusion, since more material is available, resulting in an increase of the nc-MAPbBr 3 size. In Figure 2c, we show a digital camera photograph of a series of porous $\mathrm{SiO}_{2}$ films of identical thickness infiltrated with different concentrations of $\mathrm{MAPbBr} 3$ precursors under UV irradiation. Images show a gradual change in the emitted color, perceivable by the naked eye, from the neatly green emission of the larger nanocrystals to the blue one of the smallest ones. The uniformity of the bright emission observed along the film surface also confirms the even infiltration over large areas. As the nanoparticles employed to build the scaffold are much smaller than typical visible wavelengths, the nc-MAPbBr $3 @ \mathrm{SiO}_{2}$ films are highly transparent, as it was verified by ballistic transmittance measurements ( $\mathrm{T}_{\mathrm{B}}>75 \%$ for $\lambda>510 \mathrm{~nm}$, as shown in the inset of Figure $2 \mathrm{~b}$ ). Absence of diffuse scattering makes these films also appealing for their use in screens and displays.

In order to create a film amenable to be used as an adaptable color converting coating, we took advantage of the pore volume that remained empty after embedding nc-MAPbBr 3 and load it with poly(dimethylsiloxane) (PDMS). Oligomeric precursors were first infiltrated and then polymerized at a temperature below $90^{\circ} \mathrm{C}$ in order to prevent degradation of the nanocrystals. After cooling these hybrid PDMS@nc-MAPbBr $3 @ \mathrm{SiO}_{2}$ films below the glass transition temperature of the polymer, we could lift the film off and attain a self-standing flexible film. The general scheme of the process is disclosed in Figure 3a. It is worth mentioning that this method overcomes the incompatibility between solvents used to dissolve PDMS oligomers and $\mathrm{ABX}_{3}$ perovskite precursors, since the process herein proposed do not require the dispersion of the perovskite precursors or of previously synthesized nanocrystals. We observe that encapsulation of ncMAPbBr3@SiO2 in PDMS has an advantageous effect over the photo-stability of the films. The origin of this instability in air is linked to the adsorption of negatively charged oxygen species at the materials surface, which triggers the migration of organic anions away from illuminated areas 
and through the perovskite lattice. ${ }^{26,27}$ The time dependence of the photo-emission maximum intensity is plotted in Figure $3 \mathrm{~b}$. After 10 minutes, PDMS infiltrated film preserves a bright luminescence, whereas that of bare films drops by $80 \%$ of its initial value. Remarkably, flexible films maintain both transparency (Figure 3c) and bright emission (Figure 3d) after immersion in liquid nitrogen and their subsequent lift-off. Not only PDMS provides protection against environmental agents, but also allows immersing the films in water, which has a highly deleterious effect on organic metal halide compounds. We monitored by naked eye the emission of the selfstanding PDMS@nc-MAPbBr $3 @ \mathrm{SiO}_{2}$ films when they were fully immersed in water (Figure 3e) or washed under a water-jet (Figure 3f). It can be clearly seen that the bright emission of nc$\mathrm{MAPbBr} 3$ under UV photoexcitation is kept while water is surrounding or impinging on the films. Please notice that mere exposition of $\mathrm{MAPbBr}_{3}$ to ambient humidity provokes the rapid degradation of the structure. Its adaptability is further demonstrated by using it as a color converting coating deposited onto the curved surface of a blue GaN LED. Picture of the coating after lighting the LED are shown in Figure 3g, while in Figure $3 \mathrm{~h}$ we plot the emission spectrum before and after the deposition of the flexible film on top of the LED. It can be seen that color conversion occurs in a ratio of $4: 1$, which is a value similar to that attained with other coatings devised for a similar purpose. ${ }^{28}$

In order to analyze how the emission of nc-MAPbBr 3 is affected once the pores are filled with PDMS, we measured the PL decay dynamics of both nc-MAPbBr $3 @ \mathrm{SiO}_{2}$ and PDMS@ncMAPbBr $3 @ \mathrm{SiO}_{2}$ films. A selection of the decay curves attained is displayed in a log-log plot in Figure 4a. The raw data are separately plotted in Figure S5, along with the corresponding fittings. It can be readily seen that the PL of nanocrystals decays faster as a result of the presence of PDMS in the pores. This effect is discussed next on the basis of the expected changes in the local optical 
environment of the emitters. Interestingly, analysis of the QY of both series of samples (Figure 4b) shows a maximum for films prepared using a $5 \mathrm{wt} . \%$ precursor concentration (QY $=55 \%$ for PDMS@nc-MAPbBr3@SiO 2 ; QY=36\% for nc-MAPbBr3@ $\mathrm{SiO}_{2}$ ). The occurrence of a bright point as a function of particle size has also been observed in nanocrystalline colloidal semiconductors ${ }^{29}$ as well as, in particular, in Cs-based perovskites. ${ }^{30}$ Using the expression that relates QY with the decay rates $\mathrm{QY}=\Gamma_{\mathrm{R}} /\left(\Gamma_{\mathrm{R}}+\Gamma_{\mathrm{NR}}\right)$ (being $\Gamma_{\mathrm{R}}$ and $\Gamma_{\mathrm{NR}}$ the radiative and nonradiative decay rates, respectively), we can estimate $\Gamma_{\mathrm{R}}$ and $\Gamma_{\mathrm{NR}}$ for each film. Results are shown in Figures $4 \mathrm{c}$ and $4 \mathrm{~d}$ respectively. Our analysis indicates that non-radiative decay pathways are not altered significantly by PDMS infiltration, and that improvement of QY observed is due to the enhancement of radiative pathways, as expected when the refractive index of the environment of an emitter is increased. ${ }^{31}$ In fact, we find that nanocrystals prepared at the lower concentrations (2 wt. $\%<[\mathrm{PVK}]<5$ wt. $\%$ ) show $\Gamma_{\mathrm{R} 1}=0.0177 \mathrm{~ns}^{-1}$ without PDMS and $\Gamma_{\mathrm{R} 2}=0.0434 \mathrm{~ns}^{-1}$ with PDMS infiltration. Hence $\Gamma_{\mathrm{R} 1} / \Gamma_{\mathrm{R} 2}=0.408$, which is very close to the fifth power of the ratio of the refractive indexes of the two optical environments, as predicted by the theory. ${ }^{32}$

\section{Conclusion}

In conclusion, we have demonstrated a synthetic route to attain highly emissive, transparent, flexible and environmentally stable films loaded with organic metal halide nanocrystals. Taking advantage of plane parallel and transparent matrices showing a high porosity and a narrow pore size distribution, we were able of infiltrating methylammonium lead bromide nanocrystals whose size can be precisely controlled in the range of 1.5 to $3 \mathrm{~nm}$, hence yielding green to blue luminescence due to quantum confinement effects. The materials herein presented overcome the 
processing obstacles presented by colloidal perovskite semiconductor nanocrystal suspensions and fulfill all requirements to be used as color converting coatings for LED technology. It is precisely in this field where we believe our results will open a new route for organic metal halide materials.

\section{Experimental Section}

Preparation of nanoparticle scaffolds: Colloidal dispersions of $\mathrm{SnO}_{2}$ (15\% in water, Alfa-Aesar) and $\mathrm{SiO}_{2}(34 \%$ in water, Ludox-TMA, Dupont) were purchased and diluted to $2 \%(\mathrm{w} / \mathrm{v})$ with methanol. $\mathrm{TiO}_{2}$ particles were synthesized with a method described elsewhere. ${ }^{33}$

The resulting final suspension was diluted to $2 \%(\mathrm{w} / \mathrm{v})$ in methanol. Porous nanoparticle based films were deposited by dip coating low fluorescence glass substrates (cleaned with ethanol, acetone and isopropanol) in the colloidal suspensions described before. In particular, consecutive immersions of the substrate in $\mathrm{TiO}_{2}, \mathrm{SiO}_{2}$ and $\mathrm{SnO}_{2}$ dispersions were performed for a maximum of 28, 20 and 140 times respectively, with a withdrawal speed of $100 \mathrm{~mm} / \mathrm{min}$. This method allows us to obtain layers with $1 \mu \mathrm{m}$ thickness. These matrices were stabilized at temperature of $400^{\circ} \mathrm{C}$. However, this treatment is avoided if a flexible structure is pursued. In this case the samples are heated at $\mathrm{T}=100^{\circ} \mathrm{C}$.

Synthesis of perovskite nanocrystals: The synthesis procedure of nc-ABX 3 perovskites consist on the preparation of a solution of precursors (methylammonium halide and lead halide powders in a molar ratio of 3:1) in N,N-dimethylformamide, and then the subsequent infiltration of the solution into the matrices, by means of spin coating. We prepared solutions with different concentrations by weight (from $2 \mathrm{w} \%$ to $40_{\mathrm{w}} \%$ ) of the sum of the perovskite precursors powders $\left(\mathrm{MAX}+\mathrm{PbX}_{2}\right.$ ) in DMF. By spreading the precursors through the inter-particle voids (5000 rpm for $1 \mathrm{~min}$ ) and a 
heat treatment $\left(100^{\circ} \mathrm{C}\right.$ for one hour) we promote the formation of perovskite nanocrystals that grow within these porous structures. Following an already established procedure, all the steps related to the synthesis of perovskites are conducted inside a glovebox in order to eliminate the oxygen and humidity interference and have a controllable and reproducible procedure. DMF (anhydrous, 99.8, \%Sigma Aldrich), $\mathrm{PbCl}_{2}$ (98\%, Aldrich Chemistry), $\mathrm{PbBr} 2$ (99\%, TCL), MAI (CAS:14965-49-2, Greatcell) and MABr (CAS:6876-37-5, Greatecell) have been purchased and used without further purification. Please notice that in our approach, we can skip a purification step that is required in other type of infiltration methods as impregnation, immersion, etc.

PDMS Infiltration and Preparation of Flexible Films: A solution containing a silicone elastomer and a cross-linker (Sylgard 184 elastomer kit, Dow Corning) in a mass ratio of 8 to 1 was dispersed over the porous metal oxide matrix that contains the $\mathrm{nc}-\mathrm{ABX}_{3}$ with a roller blade, then spin coated at $700 \mathrm{rpm}$ for $60 \mathrm{~s}$ and eventually heated at $90^{\circ} \mathrm{C}$ for one hour. In this way, the solution penetrates the porous structure using the remaining void space and forms a continuous layer of PDMS that embeds the MOx nanoparticles and the perovskite nanocrystals. Subsequently, we realize incisions in the edges of the sample which, after immersion in liquid nitrogen, allow the sample to be easily detached from the substrate. The whole process starting with the infiltration of PDMS and ending with lifting-off the flexible film is conducted outside the glovebox.

Optical Characterization: The analyses of the photoemission and the relative transient were conducted using a fiber-coupled spectrophotometer in which a continuous wave pump laser beam was focused with an achromatic lens producing a spot size of $250 \mu \mathrm{m}^{2}$. The excitation wavelength was selected at $450 \mathrm{~nm}$. PL decays were measured in the same optical set-up, using a pulsed light source (Fianium SC400) in concert with a time-correlated single photon counting module (FluoroHub) and de-convoluted by means of the same measuring device. Decay curves were fitted 
making use of a continuous distribution in the form of a lognormal:

$$
\rho(\Gamma)=\frac{1}{\sigma \Gamma \sqrt{2 \pi}} e^{-\frac{(\ln \Gamma-\mu)^{2}}{2 \sigma^{2}}}
$$

This distribution of the decay rates is centered in a most frequent value (mode) equal to

$$
\Gamma=e^{\left(\mu-\sigma^{2}\right)}
$$

QY measurements were carried out using the Hamamatsu C9920-02 Absolute PL Quantum Yield Measurement System that presents a Xenon lamp, a monochromator and an integration sphere. The excitation wavelength was set to $450 \mathrm{~nm}$. Photoexcitation spectra were collected using a fluorometer (Fluorolog-3 from Horiba) with a double monochromator and a spot of $1 \times 1 \mathrm{~cm}$, in a front-face configuration.

For the measurements of diffuse and direct transmittance, conducted within the visible limits, the Cary5000 spectrophotometer (UV-Vis-NIR) was used, equipped with an internal DRA-2500 (PMT / PbS version).

Structural Characterization: Samples were prepared using a focused ion beam in order to obtain lamellas that could be observed in the TEM. Then the characterization was carried out by means of a FEI Talos F200S scanning/transmission electron microscope. Images of the cross sections of the infiltrated porous matrices were obtained using a microscope (FESEM) Hitachi 5200. For these measurements, $\mathrm{SiO}_{2}$ nanoparticles were deposited on silicon substrates. XRD measurements were collected in PHILIPS X'pert PRO diffractometer. Acquisition of the samples was taken in the $5^{\circ}-50^{\circ} 2 \theta$ range with a step of $0.05^{\circ}$. 
FIGURES.
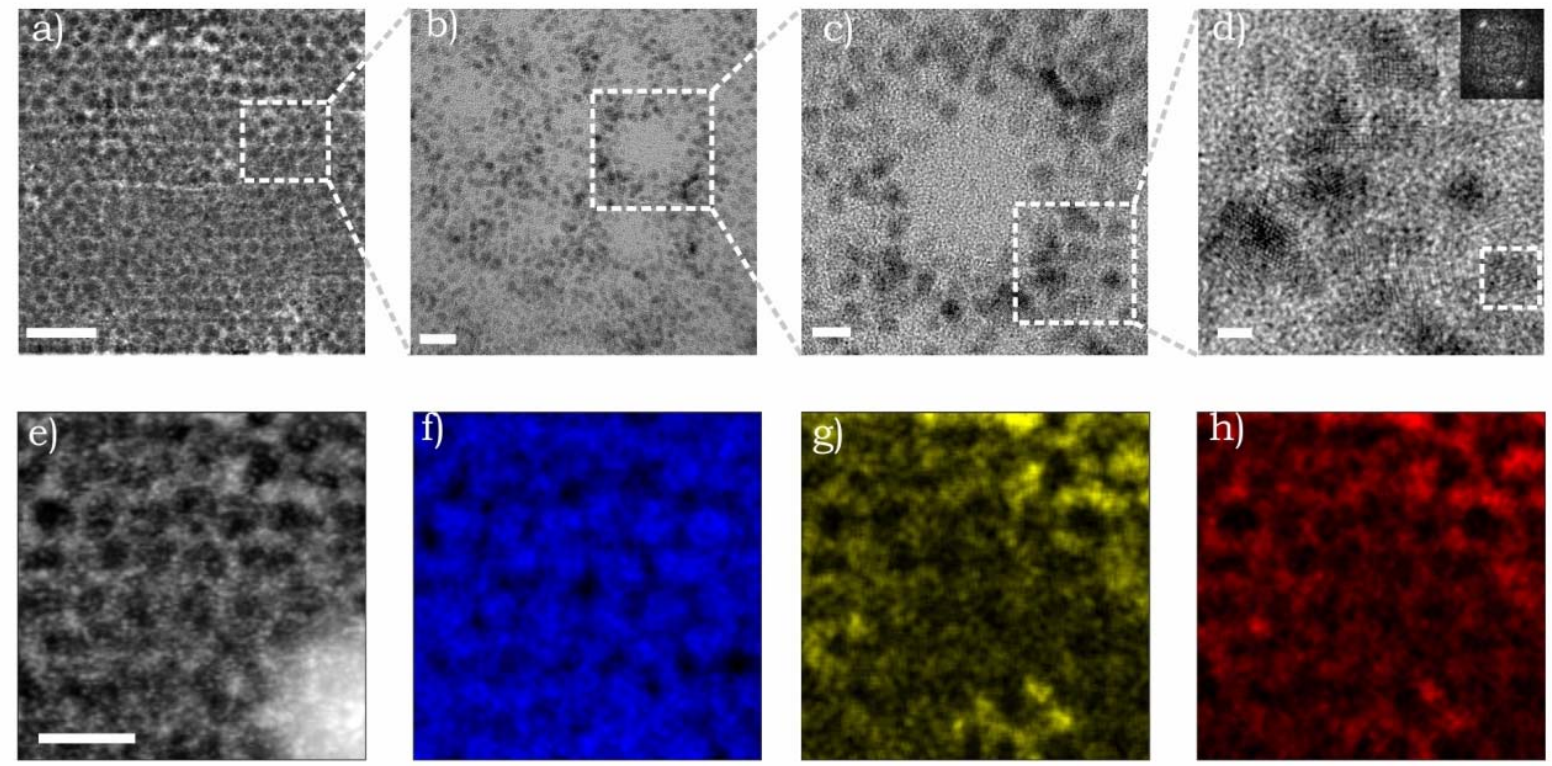

Figure 1. a) HAADF-STEM image of a cross section of a $\mathrm{SiO}_{2}$ nanoparticles mesoporous film containing nc-MAPbBr3; scale bar is $100 \mathrm{~nm}$. From b) to d) High-resolution TEM images, with different magnification of the dashed areas, of nc-MAPbBr 3 infiltrated in the voids of the mesoporous matrix; scale bar is $10 \mathrm{~nm}$ in b), $3.5 \mathrm{~nm}$ in c) and $1 \mathrm{~nm}$ in d). The inset in d) shows the digital diffraction pattern of area selected in the same panel. From f) to h), XEDS chemical mapping of the HAADF-STEM cross-section area presented in e); scale bar is $50 \mathrm{~nm}$. $\mathrm{f}$ ) Si map; g) $\mathrm{Pb}$ map; h) Br map. 

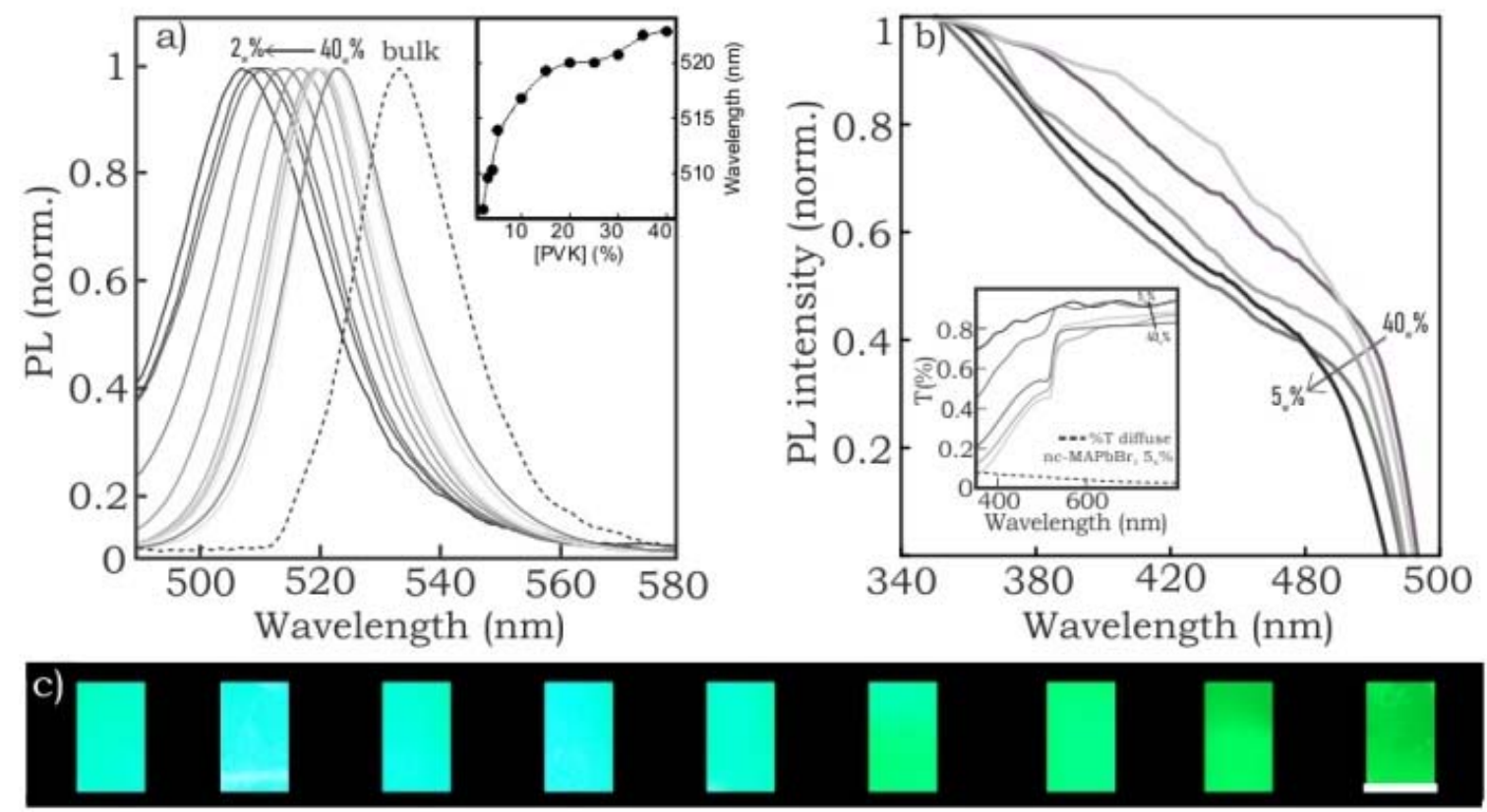

Figure 2. a) Normalized PL spectra of nc-MAPbBr3 synthesized within the voids of $\mathrm{SiO}_{2}$ scaffold, starting from solutions with different precursor concentrations (\%, $\left.\mathrm{MABr} / \mathrm{PbBr}_{2}\right)$. Gradual shift of the maximum of emission is registered from $523 \mathrm{~nm}, 40 \mathrm{wt} . \%$ to $507 \mathrm{~nm}, 2 \mathrm{wt} . \%$. The emission of bulk material is represented with black dashed line (maximum emission at 533 $\mathrm{nm}$ ). Inset: evolution of the PL maximum with the increase of perovskite precursor concentrations. b) Normalized photo-excitation spectra of nc-MAPbBr3 obtained with precursor concentration of 5 wt.\% (black solid line); 10 wt.\% (grey solid line); 20 wt.\% (silver solid line); 30 wt.\% (light grey solid line); $40 \mathrm{wt} \%$ (purple grey solid line). The inset displays the transmittance of same samples, using the same color lines and the diffuse transmittance (black dashed line) of 5\% sample. c) Photograph under UV light $(\lambda=312 \mathrm{~nm})$ of $\mathrm{nc}-\mathrm{MAPbBr} 3$ prepared (initial precursor concentration decreases from the right to the left). Scale bar is $1 \mathrm{~cm}$. 
a)
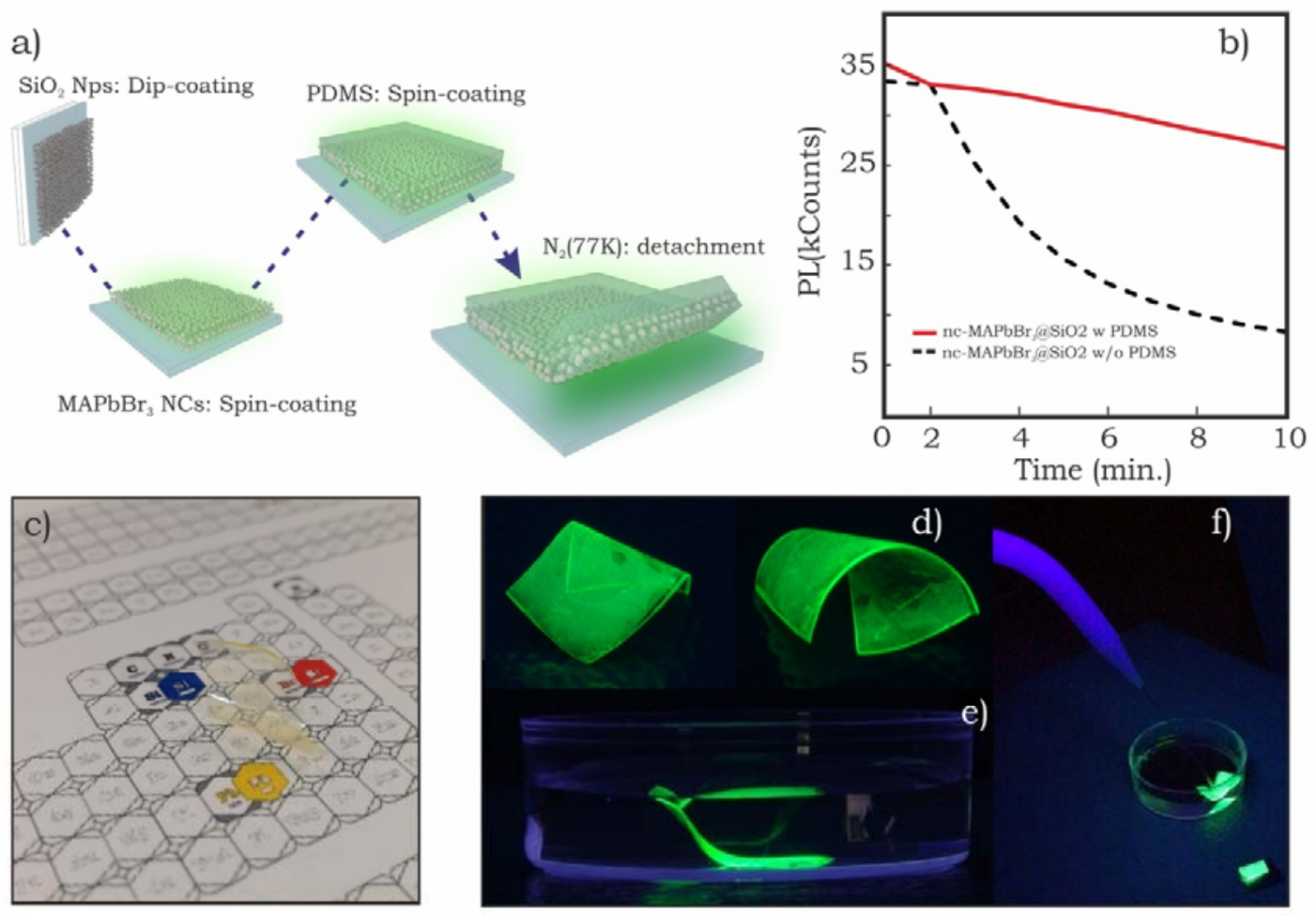

g)
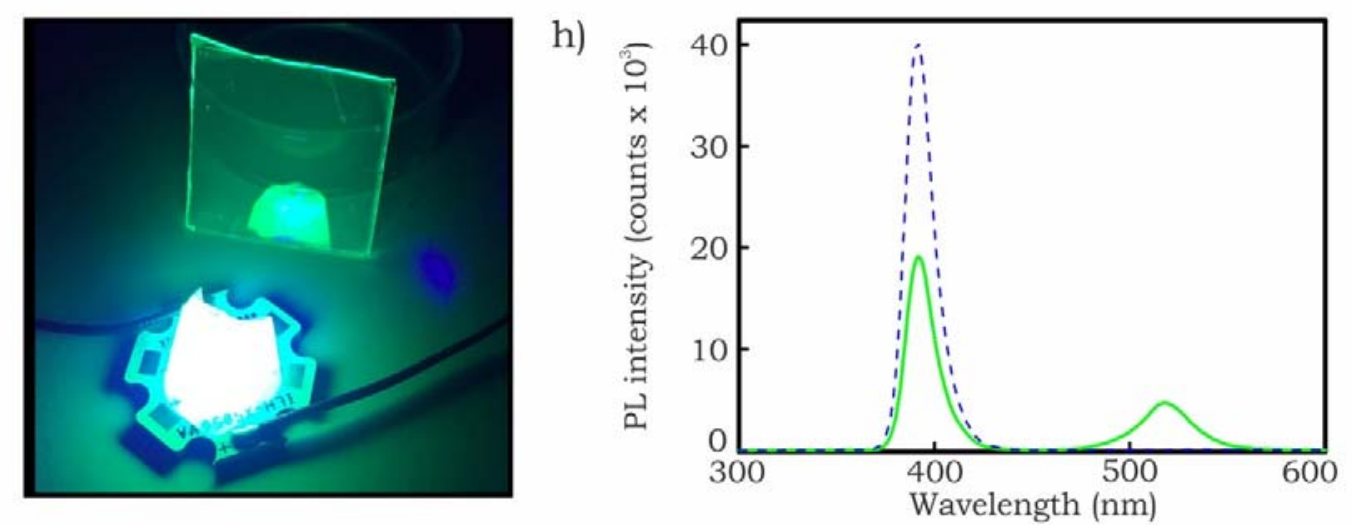

Figure 3. a) Representative scheme of the fabrication of flexible emissive film constituted by PDMS@nc-MAPbBr $3 @ \mathrm{SiO}_{2}$. b) Evolution of the photoemission of nc-MAPbBr $3 @ \mathrm{SiO}_{2}$ system (prepared from a $5 \mathrm{wt} . \%$ precursor solution) with (red solid line) and without PDMS (black dashed line).c) Daylight image of a flexible film made of PDMS@nc-MAPbBr $3 @ \mathrm{SiO}_{2}$ self-standing film. Camera pictures of flexible perovskite-silica structures exposed to UV light d), immersed in water e) and under a water jet f). g) Image of a flexible transparent film covering a 390nm LED in darkness. A glass supported nc-MAPbBr $3 \mathrm{SiO}_{2}$ is also shown at the back of the film covered LED. h) PL spectra of GaN LED before (blue dashed line) and after the deposition of flexible film made of PDMS@nc-MAPbBr3@ $\mathrm{SiO}_{2}$ (green solid line). 

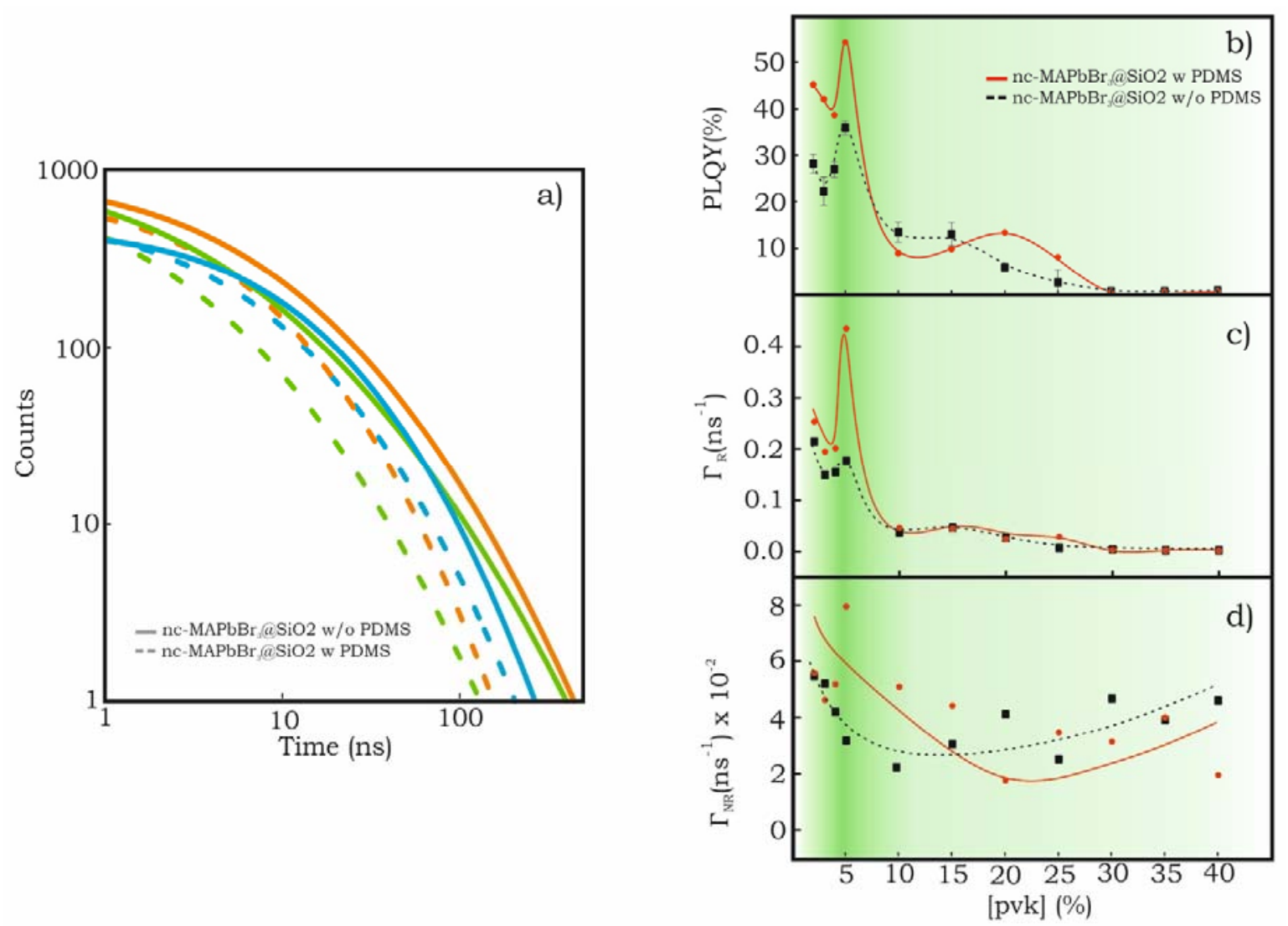

Figure 4. a)Comparison between the decay curves attained for $\mathrm{MAPbBr}_{3}$ nanocrystals, synthesized from solutions with precursor concentrations of $2 \mathrm{wt} \%$ (blue solid line), $5 \mathrm{wt} . \%$ (orange solid line) and $10 \mathrm{wt. \%}$ (green solid line), before and after being infiltrated with PDMS (dashed lines, same color code). b) Concentration-dependent QY measurements with PDMS (red circles) and without PDMS (black squares). Radiative c) and non-radiative d) decay rates calculated using the lifetimes and QY results and plotted as a function of the precursor concentration employed in the perovskite synthesis. Lines are just a guide to the eye. 


\section{ASSOCIATED CONTENT}

\section{Supporting Information.}

The following files are available free of charge.

Structural (SEM, XRD) and optical (PL, Absorptance) characterization of $\mathrm{MAPbBr}_{3}$ and

$\mathrm{MAPbI}_{3}$ nanocrystals embedded in $\mathrm{SiO}_{2}$ - and $\mathrm{TiO}_{2}$-based matrices. (PDF)

\section{AUTHOR INFORMATION}

\section{Corresponding Authors}

*Mauricio E. Calvo. E-mail: mauricio.calvo@icmse.csic.es;

*Hernán Míguez. E-mail: h.miguez@csic.es

\section{ORCID}

Mauricio E. Calvo: 0000-0002-1721-7260

Hernán Míguez: 0000-0003-2925-6360

\section{Present Addresses}

$†$ Cavendish Laboratory, Department of Physics, University of Cambridge, JJ Thomson Avenue, Cambridge CB3 0HE, UK

\section{Author Contributions}

The manuscript was written through contributions of all authors. All authors have given approval to the final version of the manuscript. 


\section{Funding Sources}

Spanish Ministry of Economy and Competitiveness grant MAT2017-88584-R;

\section{ACKNOWLEDGMENT}

Financial support of the Spanish Ministry of Economy and Competitiveness under grant MAT2017-88584-R and from the European Research Council under the European Union's Seventh Framework Programme (FP7/2007-2013/ERC Grant Agreement 307081, POLIGHT) is gratefully acknowledged. M.A. is grateful to "La Caixa" Foundation for its financial support. The authors also acknowledge the Centro de Investigación Tecnológica e Innovación (CITIUS) where structural characterizations were carried out through scanning and transmission electron microscopy and the Optoelectronic Devices Team in the Instituto de Ciencia Molecular (ICMol) of Valencia for their help with the analysis of quantum yield.

\section{REFERENCES}

(1) Kang, J.; Wang, L. W. High Defect Tolerance in Lead Halide Perovskite CsPbBr3. J. Phys.

Chem. Lett. 2017, 8, 489-493.

(2) Akkerman, Q. A.; Gandini, M.; Di Stasio, F.; Rastogi, P.; Palazon, F.; Bertoni, G.; Ball, J. M.; Prato, M.; Petrozza, A.; Manna, L. Strongly Emissive Perovskite Nanocrystal Inks for High-Voltage Solar Cells. Nat. Energy 2016, 2, 16194.

(3)Tan, Z.-K.; Moghaddam, R. S.; Lai, M. L.; Docampo, P.; Higler, R.; Deschler, F.; Price, M.;

Sadhanala, A.; Pazos, L.M.; Credgington, D.; Hanusch, F.; Bein, T,; Snaith, H. J.; Friend, R.H. 
Emitting Diodes Based on Organometal Halide Perovskite.--Supplementary Information. Nat. Nanotechnol. 2014, 9, 687-692.

(4) Cho, H.; Jeong, S.-H.; Park, M.-H.; Kim, Y.-H.; Wolf, C.; Lee, C.-L.; Heo, J. H.; Sadhanala, A.; Myoung, N.; Yoo, S.; et al. Overcoming the Electroluminescence Efficiency Limitations of Perovskite Light-Emitting Diodes. Science 2015, 350, 1222-1225.

(5) Wehrenfennig, C.; Eperon, G. E.; Johnston, M. B.; Snaith, H. J.; Herz, L. M. High Charge Carrier Mobilities and Lifetimes in Organolead Trihalide Perovskites. Adv. Mater. 2014, 26, $1584-1589$.

(6) Kim, Y.H.; Cho, H.; Lee, T.W. Metal Halide Perovskite Light Emitters PNAS 2016, 113, 11694-11702.

(7) Lozano, G. The Role of Metal Halide Perovskites in Next-Generation Lightind Devices J. Phys Chem Lett. 2018, 9, 3987-3997.

(8) Kovalenko, M.; Schaller, R.; Jarzab, D.; Loi, M. A.; Talapin, D. Inorganically Functionalized PbS-CdS Colloidal Nanocrystals: Integration into Amorphous Chalcogenide Glass and Luminescent Properties. J. Am. Chem. Soc. 2012, 134, 2457-2460.

(9) Schmidt, L. C.; Pertegás, A.; González-Carrero, S.; Malinkiewicz, O.; Agouram, S.; Mínguez Espallargas, G.; Bolink, H. J.; Galian, R. E.; Pérez-Prieto, J. Nontemplate Synthesis of $\mathrm{CH}_{3} \mathrm{NH}_{3} \mathrm{PbBr}_{3}$ Perovskite Nanoparticles. J. Am. Chem. Soc. 2014, 136, 850-853.

(10) Protesescu, L.; Yakunin, S.; Bodnarchuk, M. I.; Krieg, F.; Caputo, R.; Hendon, C. H.; Yang, R. X.; Walsh, A.; Kovalenko, M. V. Nanocrystals of Cesium Lead Halide Perovskites $\left(\mathrm{CsPbX}_{3}, \mathrm{X}=\mathrm{Cl}, \mathrm{Br}\right.$, and I): Novel Optoelectronic Materials Showing Bright Emission with Wide Color Gamut. Nano Lett. 2015, 15, 3692-3696. 
(11) Akkerman, Q. A.; D’Innocenzo, V.; Accornero, S.; Scarpellini, A.; Petrozza, A.; Prato, M.; Manna, L. Tuning the Optical Properties of Cesium Lead Halide Perovskite Nanocrystals by Anion Exchange Reactions. J. Am. Chem. Soc. 2015, 137, 10276-10281.

(12) Di Stasio, F.; Christodoulou, S.; Huo, N.; Konstantatos, G. Near-Unity Photoluminescence Quantum Yield in $\mathrm{CsPbBr}_{3}$ Nanocrystal Solid-Sate Films via Postsynthesis Treatment with Lead Bromide Chem. Mater. 2017, 29, 7663-7667.

(13) Gong, X.; Voznyy, O.; Jain, A.; Liu, W.; Sabatini, R.; Piontkowski, Z.; Walters, G.; Bappi, G.; Nokhrin, S.; Bushuyev, O.; Yuan, M.; Comin, R.; McCamant, D.; Kelley, S. O.; Sargent, E. H. Electron-Phonon Interaction in Efficient perovskite Blue Emitters Nat. Mater. 2018, 17, $550-556$.

(14) Kojima, A.; Ikegami, M.; Teshima, K.; Miyasaka, T. Highly Luminescent Lead Bromide Perovskite Nanoparticles Synthesized with Porous Alumina Media. Chem. Lett. 2012, 41, 397-399.

(15) Dirin, D. N.; Protesescu, L.; Trummer, D.; Kochetygov, I. V.; Yakunin, S.; Krumeich, F.; Stadie, N. P.; Kovalenko, M. V. Harnessing Defect-Tolerance at the Nanoscale: Highly Luminescent Lead Halide Perovskite Nanocrystals in Mesoporous Silica Matrixes. Nano Lett. 2016, 16, 5866-5874.

(16) Malgras, V.; Tominaka, S.; Ryan, J. W.; Henzie, J.; Takei, T.; Ohara, K.; Yamauchi, Y. Observation of Quantum Confinement in Monodisperse Methylammonium Lead Halide Perovskite Nanocrystals Embedded in Mesoporous Silica. J. Am. Chem. Soc. 2016, 138, $13874-13881$.

(17)Anaya, M.; Rubino, A.; Rojas, T. C.; Galisteo-López, J. F.; Calvo, M. E.; Míguez, H. Strong Quantum Confinement and Fast Photoemission Activation in $\mathrm{CH}_{3} \mathrm{NH}_{3} \mathrm{PbI}_{3}$ Perovskite 
Nanocrystals Grown within Periodically Mesostructured Films. Adv. Opt. Mater. 2017, 5, 1601087.

(18) Demchyshyn, S.; Roemer, J. M.; Groiß, H.; Heilbrunner, H.; Ulbricht, C.; Apaydin, D.; Böhm, A.; Rütt, U.; Bertram, F.; Hesser, G.; Scharber, M.C.; Sariciftci, N.S., Nickel, B.; Bauer, S.; Glowacki, E.D.; Kaltenbrunner, M. Confining Metal-Halide Perovskites in Nanoporous Thin Films. Sci. Adv. 2017, 3, e1700738.

(19) H.J. Lee, K.T. Cho, S. Paek, Y. Lee, A.J. Huckaba, V.I. E. Queloz, I. Zimmermann, G. Grancini, E. Oveisi, S.M. Yoo, S.Y. Lee, T. Shin, M. Kim, M.K. Nazeeruddin. A Facile Preparative Route of Nanoscale Perovskites over Mesoporous Metal Oxide Films and Their Applications to Photosensitizers and Light Emitters. Adv. Funct. Mater. 2018, 28, 1803801.

(20) Jiménez-Solano, A.; Galisteo-López, J. F.; Míguez, H. Fine Tuning the Emission Properties of Nanoemitters in Multilayered Structures by Deterministic Control of Their Local Photonic Environment. Small 2015, 11, 2727-2732.

(21) Calvo, M. E.; Castro Smirnov, J. R.; Míguez, H. Novel Approaches to Flexible Visible Transparent Hybrid Films for Ultraviolet Protection. J. Polym. Sci. Part B Polym. Phys. 2012, $50,945-956$.

(22) Sánchez-Sobrado, O.; Calvo, M. E.; Míguez, H. Versatility and Multifunctionality of Highly Reflecting Bragg Mirrors Based on Nanoparticle Multilayers. J. Mater. Chem. 2010, 20, 8240-8246.

(23)Tanaka, K.; Takahashi, T.; Ban, T.; Kondo, T.; Uchida, K.; Miura, N. Comparative Study on the Excitons in Lead-Halide-Based Perovskite-Type Crystals $\mathrm{CH}_{3} \mathrm{NH}_{3} \mathrm{PbBr}_{3} \mathrm{CH}_{3} \mathrm{NH}_{3} \mathrm{PbI}_{3}$. Solid State Commun. 2003, 127, 619-623. 
(24) Pack, M.; Hu, H.; Kim, D.-O.; Yang, X.; Sun, Y. Colloidal Drop Deposition on Porous Substrates: Competition among Particle Motion, Evaporation, and Infiltration. Langmuir 2015, 31, 7953-7961.

(25) Thanh, N. T. K.; Maclean, N.; Mahiddine, S. Mechanisms of Nucleation and Growth of Nanoparticles in Solution. Chem. Rev. 2014, 114, 7610-7630.

(26) Jacobsson, T. J.; Correa-Baena, J. P.; Halvani Anaraki, E.; Philippe, B.; Stranks, S. D.; Bouduban, M. E. F.; Tress, W.; Schenk, K.; Teuscher, J.; Moser, J. E.; Rensmo, H.; Hagfeldt, A. Unreacted $\mathrm{PbI}_{2}$ as a Double-Edged Sword for Enhancing the Performance of Perovskite Solar Cells. J. Am. Chem. Soc. 2016, 138, 10331-10343.

(27) Anaya, M.; Galisteo López, J.F.; Calvo, M.E., Espinós, J.P.; Míguez, H. Origin of LightInduced Photophysical Effects in Organic Metal Halide Perovskites in the Presence of Oxygen. J. Phys. Chem Lett. 2018, 9, 3891-3896.

(28) Dursun, I.; Shen, C.; Parida, M. R.; Pan, J.; Sarmah, S. P.; Priante, D.; Alyami, N.; Liu, J.; Saidaminov, M. I.; Alias, M. S.; Abdelhady, A. L.; Ng, T. K.; Mohammed, O. F.; Ooi, B. S.; Bakr, O. M. Perovskite Nanocrystals as a Color Converter for Visible Light Communication. ACS Photonics 2016, 3, 1150- 1156

(29) Qu, L.; Peng, X. Control of Photoluminescence Properties of CdSe Nanocrystals in Growth. J. Am. Chem. Soc. 2002, 124, 2049-2055.

(30) Su, Y.; Chen, X.; Ji, W.; Zeng, Q.; Ren, Z.; Su, Z.; Liu, L. Highly Controllable and Efficient Synthesis of Mixed-Halide CsPbX $3(\mathrm{X}=\mathrm{Cl}, \mathrm{Br}, \mathrm{I})$ Perovskite QDs toward the Tunability of Entire Visible Light. ACS Appl. Mater. Interfaces 2017, 9, 33020-33028. 
(31) Jiménez-Solano, A.; Galisteo-López, J.F.; Míguez, H. Absorption and Emission of Light in Optoelectronic Nanomaterials: The Role of the Local Optical Environment. J. Phys. Chem. Lett. 2018, 9, 2077-2084.

(32) Yablonovitch, E.; Gmitter, T. J.; Bhat, R. Inhibited and Enhanced Spontaneous Emission from Optically Thin AlGaAs/GaAs Double Heterostructures. Phys. Rev. Lett. 1988, 61, 25462549.

(33) Calvo, M. E.; Colodrero, S.; Rojas, T. C.; Anta, J. A.; Ocaña, M.; Míguez, H.

Photoconducting Bragg Mirrors Based on TiO2 Nanoparticle Multilayers. Adv. Funct. Mater. 2008, 18, 2708-2715.

TOC

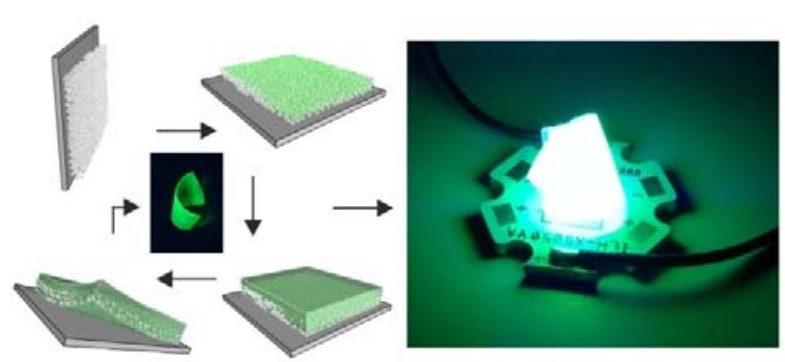


